\title{
Ordered Left Almost Hyperring
}

\author{
A M Muftirridha ${ }^{1}$, A R Alghofari ${ }^{2}$, and N Hidayat $^{2}$ \\ ${ }^{1}$ Student of Master Mathematics Study Program, Brawijaya University \\ ${ }^{2}$ Department of Mathematics, Brawijaya University \\ Email:ammufti@yahoo.com
}

\begin{abstract}
In this paper, we introduce the concept of left almost hyperring $\left(R_{,} \oplus, \otimes\right)$ together with a partial relation order $\leq$ such that satisfies some conditions. This structure $(R, \oplus, \otimes, \leq)$ is called by ordered left almsot hyperring (LA-hyperring). Further, we study some useful contiditons for ordered LA-hyperring to become an ordered hyperring. Also, we notice the notion of hyperideal, bi-hyperideal, and quasi-hyperideal of ordered LA-hyperring and their properties are investigated.
\end{abstract}

Keywords: Ordered Hyperstructure, Ordered LA-hyperring, Hyperideal, LA-hyperring.

\section{INTRODUCTION}

The algebraic hyperstructure which is a generalization theory of ordinary algebraic structures was first introduced in 1934 by a French mathematician, Marty [1]. In ordinary algebraic structure, the composition of two elements is an element, while in algebraic hyperstructure the composition of two elements is a set. Many mathematicians studied hyperstucture theory because hyperstructures have a lot of applications to many subjects of mathematics and computer science. Corsini and Leoreanu [2] discussed some applications of hyperstructure theory to geometry, hypergraphs, lattices, fuzzy sets, automata, cryptography, codes, artificial intelligence and probabilities.

In 1972, Kazim and Naseerudin [3] introduced the notion of left almost semigroup (LA-group) as a non associative group. A groupoid $(G,+)$ is said to be an LA-semigroup if satisfy $(a+b)+c=(c+b)+a$, for all $a, b, c \in G$. It is known as invertive law. Kamran [4] discussed some properties of LA-group, substructures of LA-group and the quotient structures. Later, the concept of LA-ring was introduced by Yusuf [5]. Basically, LAring correspond to ring. An algebraic structures $\left(R_{v}+, *\right)$ is a non-empty set $R$ with the binary operations " ${ }^{\text {"I }}$ and ${ }^{\mathrm{m}}{ }^{\mathrm{N}}$ such that $\left(R_{v}+\right)$ is an LA-group, $\left(R_{s}\right)$ is an LA-semigroup, both left and right distributive laws hold. There are several authors who studied LA-ring and explored some useful properties of LA-ring. Shah and Rehman [6] studied LA-ring of finitely non-zero functions which is generalize the structure of commutative semigroup ring. Hussain and Firdous [7] characterized LA-ring by the properties of their direct product.

Recently, the concept of hyperstructure was applied to LA-semigroups. It was introduced by Hila and Dine [8] as a generalization of semihypergroups and LAsemigroup. Yaqoob and Gulistan [9] introduced the notion of partially ordered LA-semihypergroup. Yaqoob et al. [10] studied intra-regular LA-semihypergroup and characterized it by using their hyperideal properties. Further, Rehman et al. [11] extended the work of Hila and Dine to algebraic hyperstructure which has two hyperoperation, that is LA-hyperring. They also characterized LA-hyperrings throught their hyperideals and hypersystems.

In this paper, we introduced the notion of ordered LA-hyperring. We established some elementary properties of ordered LA-hyperring and studied some useful conditions for ordered LA-hyperring to become an ordered hyperring. Also, we introduced some type of hyperideal of ordered LA-hyperring.

\section{PRELIMINARIES}

In this section, we recall some definitions and notions of an LA-semihypergroup, an ordered LA- 
semihypergroup, an LA-hyperring and some properties that we will use in next section.

Let $H$ be a non-empty set, then the map $\oplus: H \times H \rightarrow P^{*}(H)$ is called hyperoperation of $H$, where $P^{*}(H)$ denotes the set of all non-empty power set of $H$. A set $H$ with a hyperoperation $\oplus$ is said to be a hypergroupoid, denoted by $\left(H_{2} \oplus\right)$. If $A$ and $B$ are two subsets of $H$, the we denote

$$
\begin{aligned}
& A \oplus B=\bigcup_{\triangle \in A b \in B} a \oplus b, \\
& a \oplus B=\{a\} \oplus B_{v} \quad A \oplus b=A \oplus\{b\} .
\end{aligned}
$$

[8] A hypergroupoid $\left(H_{2} \oplus\right)$ is said to be an LAhypersemigroup if satisfies left invertive law. If $\left(H_{0} \oplus\right)$ satisfies reproduction law, that is $H \oplus a=H=a \oplus H$, for every $a \in H$, then $(H, \oplus)$ is called by LAhypergroup. An element $e \in H$ is called by a left identity element (resp. pure left identity element) if $a \in a \oplus e(a=a \oplus e)$. In an LA-semihypergroup, the medial law holds, $(a \oplus b) \oplus(c \oplus d)=(a \oplus c) \oplus(b \oplus d)$ for all $a, b, c, d \in H$. An LA-semihypergroup may not contain a left identity element or a pure left identity. In an LAsemihypergroup with pure left identity, the paramedial law $(a \oplus b) \oplus(c \oplus d)=(d \oplus c) \oplus(b \oplus a)$ holds for all $a_{s} b, c_{v} d \in H$.

Definition 2.1 [9] An ordered LA-semihypergroup $\left(H_{v} \oplus, \subseteq\right)$ is a poset $\left(H_{s} \leq\right)$ at the same time an LAsemihyepergroup $(H, \oplus)$ such that $a, b, x \in H_{x} a \leq b$ implies $a \oplus x \leq b \oplus x$ and $x \oplus a \leq x \oplus b$ for any $a, b, x \in H$. A non-empty subset $A$ of $H$ is called by LAsubsemihypergroup of an ordered LAsubsemihypergroup $(H, \oplus, \leq)$ if $(A \oplus A] \subseteq(A]$.

If $A$ and $B$ are non-empty subsets of $H$, then we denote $A \leq B$ if for every $a \in B$ there exist $b \in B$ such that $a \leq b$.

Definition 2.2 [9] A non-empty subset $A$ of an ordered LA-subsemihypergroup $\left(H_{0} \oplus, \leq\right)$ is called left (resp. right) hyperideal of $\left(H_{0} \oplus, \leq\right)$ if the following conditions hold:

- $H \oplus A \subseteq A$ (resp. $A \oplus H \subseteq A$ ).

- If $a \in A$ and $b \leq a$, then $b \in A$.

$A$ is called by hyperideal of $\left(H_{0} \oplus, \leq\right)$ if it is a left and right hyperideal.

Definition 2.3 [9] An LA-subsemihypergroup $B$ of an ordered LA-semihypergroup $\left(H_{v} \oplus_{s} \leq\right)$ is called a bihyperideal of $\left(H_{v} \oplus, \leq\right)$ if the following conditions hold:

- $(B \oplus H) \oplus B \subseteq B$.

- If $a \in B$ and $b \leq a$, then $b \in B$.

Definition 2.4 [9] A non-empty subset $Q$ of an LAsemihyperring $\left(H_{0} \oplus, \leq\right)$ is called by quasi-hyperideal of $\left(H_{2} \oplus, \leq\right)$ if the following conditions hold:

- $Q \oplus H \cap H \oplus Q \subseteq Q$.

- If $a \in Q$ and $b \leq a$, then $b \in Q$.
Definition 2.5 [11] A hypergroupoid $\left(R_{v} \oplus, \otimes, \leq\right)$ is said to be an LA-hyperring if satisfies the following conditions:

- $\left(R_{v} \oplus\right)$ is an LA-hypergroup.

- $(R, \otimes)$ is an LA-hypersemigroup.

- The hyperoperation $\otimes$ is distributive with respect to the hyperoperation $\oplus$.

Definition 2.6 [11] An LA-subhypergroup $S$ of an ordered LA-hyperring $\left(R_{v} \oplus, \otimes, \leq\right)$ is said to be a left (resp. right) hyperideal of $R$ if $R \otimes S \subseteq S(S \otimes R \subseteq S)$. If $S$ is a left and right hyperideal, then $S$ is called by hyperideal.

Proposition 2.7 If $(R, \oplus, \otimes)$ be an LA-hyperring with left identity (pure left identity), then every right hyperideal is a left hyperideal.

\section{ORDERED LA-HYPERRING}

In this section, we introduce the concept of ordered LA-hyperring and give some examples of this hyperstructure. We also prove the elementary properties of ordered LA-hyperring and study some useful contiditons for ordered LA-hyperring to become an ordered hyperring.

Definition 3.1 A hypergroupiod $\left(R_{v} \oplus, \otimes, \leq\right)$ is said to be a ordered LA-hyperring if satistfies the following conditions:

- $(R, \oplus, \otimes)$ is an LA-hyperring.

- If $a \leq b$, then $a \oplus c \leq b \oplus c$ and $c \oplus a \leq c \oplus b$.

- If $a \leq b$, then $a \otimes c \leq b \otimes c$ and $c \otimes a \leq c \otimes b$.

Example 3.2 Let $R=\{a, b, c, d\}$ be a set with the hyperoperations $\oplus$ and $\otimes$ are defined as follows:

Table 1. The hyperoperation $\oplus$ of LA-hyperring

\begin{tabular}{|l|l|l|l|l|}
\hline$\oplus$ & $a$ & $b$ & $\boldsymbol{c}$ & $\boldsymbol{d}$ \\
\hline $\boldsymbol{a}$ & $\{a\}$ & $\{a, b\}$ & $R$ & $R$ \\
\hline $\boldsymbol{b}$ & $\{a, b\}$ & $\{a, b\}$ & $R$ & $\left\{c_{x} d\right\}$ \\
\hline $\boldsymbol{c}$ & $R$ & $\left\{c_{x} d\right\}$ & $\left\{c_{x} d\right\}$ & $\left\{c_{v} d\right\}$ \\
\hline $\boldsymbol{d}$ & $R$ & $R$ & $R$ & $R$ \\
\hline
\end{tabular}

Table 2. The hyperoperation $\otimes$ of LA-hyperring

\begin{tabular}{|l|l|l|l|l|}
\hline$\otimes$ & $a$ & $b$ & $\boldsymbol{c}$ & $\boldsymbol{d}$ \\
\hline $\boldsymbol{a}$ & $\{a\}$ & $\{a\}$ & $\{a\}$ & $\{a\}$ \\
\hline $\boldsymbol{b}$ & $\{a\}$ & $\{a, b\}$ & $R$ & $R$ \\
\hline $\boldsymbol{c}$ & $\{a\}$ & $R$ & $R$ & $\{d\}$ \\
\hline
\end{tabular}




\begin{tabular}{|l|l|l|l|l|}
\hline $\boldsymbol{d}$ & $\{a\}$ & $R$ & $\{c, d\}$ & $R$ \\
\hline
\end{tabular}

And the relation order $\leq$ is defined by: $\leq=$

$\{(a, b),(b, d),(c, d),(a, d),(a, a),(b, b),(c, c),(d, d)\}$

It is easy to verify that $(R, \oplus, \otimes, \subseteq)$ is an ordered LAhyperring and we can see that $\left(R_{s} \oplus\right)$ and $\left(R_{s} \otimes\right)$ are non-associative,

$(c \oplus b) \oplus d=R \neq\{c, d\}=c \oplus(b \oplus d)$

and

$(c \otimes c) \otimes d=R \neq\{d\}=c \otimes(c \otimes d)$.

Definition 3.3 Let $(R, \oplus, \otimes, \leq)$ be an ordered LAhyperring and $A \subseteq R$. Define a subset $(A]$ as follows $(A]=\{t \in R: t \leq a$, for some $a \in A\}$

Lemma 3.4 Let $(R, \oplus, \otimes, \subseteq)$ be an ordered LAhyperring. Then

- $A \subseteq(A]$ for any $A \subseteq R$.

- If $A \subseteq B$, then $(A] \subseteq$ (B] for any $A_{v} B \subseteq R$.

- $(A] \otimes(B] \subseteq(A \otimes B]$ and $(A] \oplus(B] \subseteq(A \oplus B]$ for any $A_{s} B \subseteq R$.

- $\quad(A] \otimes(B]]=(A \otimes B]$ and $(C A] \oplus(B]]=(A \oplus B]$ for any $A_{x} B \subseteq R$.

- If $A \subseteq B$, then $(C \otimes A] \subseteq(C \otimes B]$ and $(A \otimes C] \subseteq(B \otimes C]$.

- If $A \subseteq B$, then $(C \oplus A] \subseteq(C \oplus B]$ and $(A \oplus C] \subseteq(B \oplus C]$.

Proof. The proof is straightforward.

Theorem 3.5 An ordered LA-hyperring $(R, \oplus, \otimes, \subseteq)$ is an ordered hyperring if and only if:

- $a \oplus b=b \oplus a$.

- $a \otimes(b \otimes c)=(c \otimes b) \otimes a$.

Proof. Let $(R, \oplus, \otimes, \leq)$ be an ordered LA-hyperring. We will show that $(R, \oplus, \otimes, \leq)$ is an ordered hyperring. Let $a, b, c \in R$, then

$(a \oplus b) \oplus c=(c \oplus b) \oplus a \quad$ (left invertive law)

$$
=a \oplus(b \oplus c)
$$

Since $\left(R_{s} \oplus\right)$ is a LA-hypergroup, so reproduction axioms holds in $R$. Thus $(R, \oplus)$ is a hypergroup.

$$
\begin{aligned}
(a \otimes b) \otimes c & =(c \otimes b) \otimes a \\
& =a \otimes(b \otimes c)
\end{aligned}
$$

Hence, $\left(R_{2} \oplus, \otimes\right)$ is a hyperring. Since $R$ is an ordered LA-hyperring, the conditions (R1) and (R2) holds obviously. So $(R, \oplus, \otimes, \subseteq)$ is an ordered hyperring.

Definition 3.6 Let $\left(R_{v} \oplus, \otimes, \leq\right)$ is an ordered LAhyperring, then:

- $R$ is called with left identity (resp. pure left identity) if there is an element $e \in R$ such that $a \in a \otimes e$ $(\{a\}=a \otimes e)$ for all $a \in R$.
- A non-empty subset $S$ of $R$ is said to be an LAsubsemihyperring if $(s, \oplus, \otimes, \leq)$ itself is an ordered LA-hyperring.

- An element $a \in R$ is called by an idempotent (resp. weakly idempotent) element of $R$ if $a \otimes a=a$ (resp. $a \otimes a=\{a\}$ ) .

Example 3.7 Let $A=\{a, b, c\}$ with the hyperoperations $\oplus$ and $\otimes$ are defined as follows:

Table 3. The hyperoperation $\oplus$ of $A$.

\begin{tabular}{|c|c|c|c|}
\hline$\oplus$ & $\boldsymbol{a}$ & $\boldsymbol{b}$ & $\boldsymbol{c}$ \\
\hline $\boldsymbol{a}$ & $s$ & $s$ & $s$ \\
\hline $\boldsymbol{b}$ & $\{a, b\}$ & $\{b, c\}$ & $\{b, c\}$ \\
\hline $\boldsymbol{c}$ & $\{a, c\}$ & $\{b, c\}$ & $\{b, c\}$ \\
\hline
\end{tabular}

Table 4. The hyperoperation $\oplus$ of $A$.

\begin{tabular}{|c|c|c|c|}
\hline$\otimes$ & $\boldsymbol{a}$ & $\boldsymbol{b}$ & $\boldsymbol{c}$ \\
\hline $\boldsymbol{a}$ & $\{a\}$ & $\{b\}$ & $\{c\}$ \\
\hline $\boldsymbol{b}$ & $\{c\}$ & $\{b, c\}$ & $\{c\}$ \\
\hline $\boldsymbol{c}$ & $\{b\}$ & $\{b\}$ & $\{b, c\}$ \\
\hline
\end{tabular}

And the order relation is defined by $\leq:=\{(a, b),(a, c),(a, a),(b, b),(c, c)\}$.

It is easy to verify that $\left(A_{2} \oplus, \otimes, \leq\right)$ is an ordered LAhyperring and $a$ is a pure identity element of $A$.

Theorem 3.8 A pure left element of an ordered LAhyperring is unique.

Proof. Let $(R, \oplus, \otimes, \subseteq)$ be an ordered LA-hyperring and $e$ be a pure left identity element. Assume that a pure left identity is not unique, then there is an element $e^{\prime} \in R$ such that $\left\{e^{\prime}\right\}=e^{\prime} \otimes a$, for all $a \in R$.

$$
\{e\}=e \otimes e^{s}
$$

$$
\begin{array}{ll}
=(e \otimes e) \otimes e^{v} & (e \text { is a pure left identity) } \\
=\left(e^{v} \otimes e\right) \otimes e & \text { (invertive law) } \\
=e^{v} \otimes e=\left\{e^{v}\right\} &
\end{array}
$$

It contradicts with the assumption that $e$ is not unique. Hence a pure left identity is unique.

\section{HYPERIDEAL OF ORDERED LA- HYPERRING}

In this section, we study hyperideal, bi-hyperideal, and quasi-hyperideal of ordered LA-hyperring. Also we investigate some elementary properties of some type hyperideal of ordered LA-hyperring.

Definisi 4.1 A non-empty set $I$ of an ordered LAhyperring $(R, \oplus, \otimes, \leq)$ is called by left (resp. right) 
hyperideal of $\left(R_{v} \oplus, \otimes, \subseteq\right)$ if satisfies the following condition.

- $(I, \oplus)$ is LA-subhypergroup of $(R, \oplus)$.

- $R \otimes I \subseteq I(I \otimes R \subseteq I)$.

- If $a \in I$ and $b \leq a$, then $b \in I$, for any $b \in R$.

Example 4.2 Let $(R, \oplus, \otimes, \subseteq)$ be an ordered LAhyperring with the hyperoperations $\oplus$ and $\otimes$ are defined as follows:

Table 5. The hyperoperation $\oplus$ of $R$.

\begin{tabular}{|c|c|c|c|}
\hline$\oplus$ & $a$ & $b$ & $c$ \\
\hline$a$ & $R$ & $R$ & $R$ \\
\hline$b$ & $\{a, b\}$ & $\{b, c\}$ & $\{b, c\}$ \\
\hline$c$ & $\{a, c\}$ & $\{b, c\}$ & $\{b, c\}$ \\
\hline
\end{tabular}

Table 6. The hyperoperation $\otimes$ of $R$.

\begin{tabular}{|c|c|c|c|}
\hline$\otimes$ & $a$ & $b$ & $c$ \\
\hline$a$ & $R$ & $\{b, c\}$ & $\{b, c\}$ \\
\hline$b$ & $\{b, c\}$ & $\{b, c\}$ & $\{c\}$ \\
\hline$c$ & $\{b, c\}$ & $\{b\}$ & $\{b, c\}$ \\
\hline
\end{tabular}

And the relation order is defined by $\leq:=\{(a, a),(b, b),(c, c),(b, a),(c, a)\}$.

It is easy to verify that $l=\{b, c\}$ is a hyperideal of $(R, \oplus, \otimes, \leq)$.

Teorema 4.3 The intersection of any two hyperideals of ordered LA-hyperring $\left(R_{*} \oplus, \otimes, \leq\right)$ is a hyperideal of $R$.

Proof. Let $I_{1}$ and $I_{2}$ be two hyperideals of $R$. First, we will show that $I_{1} \cap I_{2}$ is an LA-subhypergroup of $(R, \oplus)$. Let $\quad x, y \in I_{1} \cap I_{2}$, then we have $x \oplus y \subseteq I_{1} \oplus I_{1} \subseteq I_{1}$ and $x \oplus y \subseteq I_{2} \oplus I_{2} \subseteq I_{2}$. Since $x \oplus y \subseteq I_{1}, x \oplus y \subseteq I_{2}$ and $I_{1}, I_{2}$ are hyperideal of $R$, then the left invertive law is satisfied in $I_{1} \cap I_{2}$. By definition of hyperideal, $I_{1}$ and $I_{2}$ are LAsubhypergroup of $\left(R_{s} \oplus\right)$, then for any $r \in R$ and $x \in I_{1} \cap I_{2}$ we get $r \oplus I_{1} \cap I_{2} \subseteq r \oplus I_{1}=I_{1}$ and $r \oplus I_{1} \cap I_{2} \subseteq r \oplus I_{2}=I_{2}$. Conversely, $I_{1} \cap I_{2} \subseteq I_{1}=r \oplus I_{1}$ and $I_{1} \cap I_{2} \subseteq I_{2}=r \oplus I_{2}$. The same way can used to show that $I_{1} \cap I_{2} \oplus r=I_{1} \cap I_{2}$. Therefore, $I_{1} \cap I_{2}$ is an LA-subhypergroup of $\left(R_{s} \oplus\right)$.

Now we will show that $\left(I_{1} \cap I_{2}\right)$ satisfy (I2) conditions. Consider $\quad\left(I_{1} \cap I_{2}\right) \otimes R \subseteq I_{1} \otimes R \subseteq I_{1} \quad$ and $\left(I_{1} \cap I_{2}\right) \otimes R \subseteq I_{2} \otimes R \subseteq I_{2}$. This implies that $\left(I_{1} \cap I_{2}\right) \otimes R \subseteq I_{1} \cap I_{2}$. The case for right hyperideal can be seen in the similar way. Since $I_{1}$ and $I_{2}$ are hyperideals of $R$, then the third condition holds obviously. So $I_{1} \cap I_{1}$ is a hyperideal of $R$.
Theorem 4.4 If $\left(R_{s} \oplus, \otimes, \leq\right)$ be an ordered LAhyperring with a pure left identity $e$, then for all $a \in R$ :

- $\quad(R \otimes a$ ] is a left hyperideal of $R$.

- $\quad(a \otimes R]$ is a right hyperideal of $R$.

Proof. Let $\{x\},\{y\} \subseteq(R \otimes a]$ where $\{x\} \subseteq r_{1} \otimes a$ and $\{y\} \subseteq r_{2} \otimes a$ for some $r_{1}, r_{2} \in R$. This implies that $r_{1} \otimes a \leq s_{1} \otimes a$ and $r_{2} \otimes a \leq s_{2} \otimes a$ for some $s_{1}, s_{2} \in R$.

$\{x\} \oplus\{y\} \subseteq\left(r_{1} \otimes a\right) \oplus\left(r_{2} \otimes a\right)$

$\leq\left(s_{1} \otimes a\right) \oplus\left(s_{2} \otimes a\right)$

$=\left(s_{1} \oplus s_{2}\right) \otimes a \subseteq R \otimes a$

Thus $\{x\} \oplus\{y\} \subseteq(R \otimes a]$. For any $r \in R$, we have

$\{x\} \oplus(R \otimes a] \subseteq r_{1} \otimes a \oplus(R \otimes a]$

$\leq r_{1} \otimes a \oplus r \otimes a$

$=\left(\sigma_{1} \oplus r\right) \otimes a \subseteq R \otimes a$

And

$\{x\} \subseteq r_{1} \otimes a \leq s_{1} \otimes a$

$\subseteq R \otimes a$

$=(r \oplus R) \otimes a$

$=(r \otimes a) \oplus(R \otimes a)$

Hence $\{x\} \oplus(R \otimes a]=(R \otimes a]$. The similar way can be used to show that $(R \otimes a] \oplus\{x\}=(R \otimes a]$. Therefore, $(R \otimes a]$ is an LA-subhypergroup of $R$.

Now let $r \in R$, then

$r \otimes\{x\} \leq r \otimes\left(r_{1} \otimes a\right)$

$$
\begin{aligned}
& \subseteq(e \otimes r) \otimes\left(r_{1} \otimes a\right) \quad \text { (pure left identity) } \\
& =\left(a \otimes r_{1}\right) \otimes(r \otimes e) \quad \text { (paramedial law) } \\
& =\left(a \otimes r_{1}\right) \otimes r \quad \text { (pure left identity) } \\
& =\left(r \otimes r_{1}\right) \otimes a \quad \text { (medial law) } \\
& \subseteq R \otimes a
\end{aligned}
$$

So $(R \otimes a]$ is a hyperideal of $R$.

Theorem 4.5 Let $(R, \oplus, \otimes, \subseteq)$ be an ordered LAhyperring with a pure left identity. If $I$ is a hyperideal of $R$, then $[\because \otimes I]$ is also a hyperideal of $R$.

Proof. First we show that $(A \otimes A]$ is an LAsubhypergroup of $\left(R_{0} \oplus, \otimes, \leq\right)$. Let $\{x\},\{y\} \subseteq(I \otimes I]$, which implies that $\{x\} \leq x_{1} \otimes x_{2}$ and $\{y\} \leq y_{1} \otimes y_{2}$ for some $x_{1} \otimes x_{2}, y_{1} \otimes y_{2} \subseteq I \otimes I$. Then we have $\{x\} \oplus\{y\} \leq x_{1} \otimes x_{2} \oplus y_{1} \otimes y_{2} \subseteq I \oplus I . \quad$ Next we show that $(I \otimes I]$ satisfy the reproduction law. Let $\{a\} \subseteq z \oplus(I \otimes I]$ then $\{a\} \subseteq\{z\} \oplus\{i\} \quad$ where $\{z\},\{i\} \subseteq(I \otimes I]$. By definition of $(I \otimes I]$, we have $\{z\} \leq z_{1} \otimes z_{2}$ and $i \leq i_{1} \otimes i_{2}$ for some $z_{1}, z_{2}, i_{1}, i_{2} \in I$. Then
$\{a\} \subseteq\{a\} \oplus\{i\}$
$\leq z_{1} \otimes z_{2} \oplus i_{1} \otimes i_{2}$

$$
\subseteq I \oplus I
$$
And

$$
\begin{aligned}
\{i\} & \leq i_{1} \otimes i_{2} \subseteq i_{1} \otimes I \\
& =i_{1} \otimes(b \oplus I) \\
& =\left(i_{1} \otimes b\right) \oplus\left(i_{1} \otimes I\right)
\end{aligned}
$$
(for all $b \in I$ ) 


$$
\subseteq\left(i_{1} \otimes b\right) \oplus(I \otimes I)
$$

Thus $(I \otimes I]$ is an LA-subhypergroup of $\left(R_{,} \oplus\right)$. Let $\{x\} \subseteq R \otimes(I \otimes I]$ where $\{x\} \subseteq r \otimes\left(i_{1} \otimes i_{2}\right)$. This implies that $i_{1} \otimes i_{2} \leq y \otimes z$ for some $y_{s} z \in I$. We have

$$
\begin{aligned}
\{x\} & \subseteq r \otimes\left(i_{1} \otimes i_{2}\right) & & \\
& \leq r \otimes(y \otimes z) & & \\
& =(e \otimes r) \otimes(y \otimes z) & & \text { (e is a pure identity) } \\
& =\left(e \otimes i_{1}\right) \otimes\left(r \otimes i_{2}\right) & & \text { (medial law) } \\
& \subseteq(I \otimes I) & &
\end{aligned}
$$

The case for $\{x\} \subseteq(I \otimes I] \otimes R$ can be seen in similar way. Let $\{x\} \subseteq(I \otimes I]$, then $\{x\} \leq x_{1} \otimes x_{2}$ for some $x_{1}, x_{2} \in I$. If $\{y\} \leq\{x\}$, then $\{y\} \leq\{x\} \leq x_{1} \otimes x_{2}$. Hence $\{y\} \subseteq(I \otimes I]$. So $[I \otimes I]$ is a hyperideal of $R$.

Definition 4.6 A non-empty set $B$ of an ordered LAhyperring $(R, \oplus, \otimes, \subseteq)$ is called by bi-hyperideal of $(R, \oplus, \otimes, \subseteq)$ if satisfies the following condition.

- $(B, \oplus)$ is LA-subhypergroup of $\left(R_{v} \oplus\right)$.

- $(B \otimes R) \otimes B \subseteq B$.

- If $a \in B$ and $b \leq a$, then $b \in B$, for any $b \in R$.

Theorem 4.7 Let $(R, \oplus, \otimes, \leq)$ be an ordered LAhyperring with a pure left identity $e$. If $B_{1}$ and $B_{2}$ are bihyperideals of $R$, then $\left(B_{1} \otimes B_{2}\right.$ ] is a bi-hyperideal of $R$.

Proof. The proof is straightforward.

Theorem 4.8 If $\left(R_{s} \oplus, \otimes, \leq\right)$ is an ordered LAhyperring, then every left (right) hyperideal of $R$ is a bihyperideal of $R$.

Proof. Let $I$ be a left hyperideal of $R$. we will show that $I$ satisfy the (B2) conditions.

$(I \otimes R) \otimes I=(R \otimes R) \otimes I \subseteq R \otimes I$.

Since $I$ is a left hyperideal of $R$, we have $R \otimes I \subseteq I$.

The case for a right hyperideal $I$ of $R$, we get

$(I \otimes R) \otimes I \subseteq I \otimes I \subseteq I$. So, $I$ is a bi-hyperideal of $R$.

Definition 4.9 A non-empty set $Q$ of an ordered LAhyperring $(R, \oplus, \otimes, \leq)$ is called by quasi-hyperideal of $(R, \oplus, \otimes, \leq)$ if satisfies the following condition.

- $(Q, \oplus)$ is LA-subhypergroup of $(Q, \oplus)$.

- $(Q \otimes R) \cap(R \otimes Q) \subseteq Q$.

- If $a \in Q$ and $b \leq a$, then $b \in Q$, for any $b \in R$.

Theorem 4.10 Let $(R, \oplus, \otimes, \subseteq)$ be an ordered LAhyperring. If $Q$ is a quasi-hyperideal of $R$, then $Q$ is an LA-subhyperring of $R$.

Proof. We will show that $Q \otimes Q \subseteq Q$. Let $x, y \in Q$, then

$x \otimes y \subseteq Q \otimes Q \subseteq Q \otimes R$

$x \otimes y \subseteq Q \otimes Q \subseteq R \otimes Q$.

and

$x \otimes y \subseteq(Q \otimes R) \cap(R \otimes Q)$. By definition of quasihyperideal of $R$, we get $x \otimes y \subseteq Q$. So, $Q$ is an LAsubhyperring of $R$.
Theorem 4.11 If $\left(R_{2} \oplus, \otimes, \leq\right)$ is an ordered LAhyperring with a pure left identity, then every quasihyperideal of $R$ is a bi-hyperideal of $R$.

Proof. Let $e$ be a pure left identity of $R$ and $Q$ be a quasi-hyperideal of $R$. We will show that $Q$ satisfy the condition $(Q \otimes R) \otimes Q \subseteq Q$. Then

$(Q \otimes R) \otimes Q \subseteq(Q \otimes R) \otimes(Q \otimes e)$

$$
=(Q \otimes Q) \otimes(R \otimes e)
$$

$$
\subseteq Q \otimes R
$$

and

$$
\begin{aligned}
(Q \otimes R) \otimes Q & \subseteq(R \otimes R) \otimes Q \\
& \subseteq R \otimes Q
\end{aligned}
$$

Hence, we get $(Q \otimes R) \otimes Q \subseteq Q \otimes R \cap R \otimes Q \subseteq Q$.

So, $Q$ is a bi-hyperideal of $R$.

\section{CONCLUSION}

An ordered LA-hyperring is a hyperstructure with a partial order relation as a generalization of LA-ring and hyperring. We obtained some elementary properties of ordered LA-hyperring and some useful contiditons for ordered LA-hyperring to become an ordered hyperring. Also, we investigated some properties of hyperideal, bihyperideal, and quasi-hyperideal of ordered LAhyperring.

\section{AUTHORS' CONTRIBUTIONS}

All authors have equally contributed to this work.

\section{ACKNOWLEDGMENTS}

We would like to thank to all referees for valuable comments. The comments and suggestions have really helped in the development of this paper.

\section{REFERENCES}

[1] MARTY and F., "Sur une generalization de la notion de groupe," 8th Congr. Math. Scandinaves, pp. 45-49, 1934.

[2] P. Corsini and V. Leoreanu, Applications of Hyperstructure Theory. Dordrecht: Kluwer Academic, 2003.

[3] M. A. Kazim and N. Naseeruddin, "On almost semigroups,” Aligarh Bull. Math., vol. 2, pp. 17, 1972.

[4] M. S. Kamran, "Conditions for LA-semigroup to resemble associative structures," Quad-iAzam University, 1993.

[5] S. M. Yusuf, “On the Left Almost Ring," Proc. 7th Int. Pure Math. Conf., 2006.

[6] T. Shah, "On LA-Rings of Finitely Nonzero Functions," vol. 5, no. 5, pp. 209-222, 2010. 
[7] F. Hussain, S. Firdous, and N. Sadiq, "Direct Product of two Near Left Almost Rings," Int. J. Algebr. Stat., vol. 5, no. 2, p. 77, 2016.

[8] K. Hila and J. Dine, "On Hyperideals in Left Almost Semihypergroups," ISRN Algebr., vol. 2011, pp. 1-8, 2011.

[9] N. Yaqoob and M. Gulistan, "Partially ordered left almost semihypergroups," J. Egypt. Math. Soc., vol. 23, no. 2, pp. 231-235, 2015.

[10] N. Yaqoob, P. Corsini, and F. Yousafzai, “On intra-regular left almost semihypergroups with pure left identity," J. Math., vol. 2013, 2013.

[11] I. Rehman, N. Yaqoob, and S. Nawaz, "Hyperideals and hypersystems in LAhyperrings," Songklanakarin J. Sci. Technol., vol. 39, no. 5, pp. 651-657, 2017. 